\title{
Conscience et méconnaissance dans Edward II de Marlowe
}

\section{Ann Lecercle}

Marie-Thérèse Jones-Davies (éd.)

\section{OpenEdition \\ Journals}

\section{Édition électronique}

URL : http://journals.openedition.org/shakespeare/440

DOI : $10.4000 /$ shakespeare.440

ISSN : 2271-6424

\section{Éditeur}

Société Française Shakespeare

\section{Édition imprimée}

Date de publication : 1 novembre 1981

Pagination : 115-132

ISBN : 2-86433-016-4

Référence électronique

Ann Lecercle, "Conscience et méconnaissance dans Edward // de Marlowe », Actes des congrès de la Société française Shakespeare [En ligne], 3 | 1981, mis en ligne le 01 novembre 2007, consulté le 20 janvier 2020. URL : http://journals.openedition.org/shakespeare/440 ; DOI : 10.4000/shakespeare.440 


\title{
THEATRE ET IDEOLOGIES :
} Marlowe, Shakespeare

\author{
DIRECTEUR DE LA PUBLICATION \\ M.T. Jones - Davies
}

JEAN TOUZOT Libraire - Editeur

38 , rue Saint-Sulpice 75278 PARIS CEDEX 061982 
1982 Jean Touzot Libiaire-Editeur, Paris.

Reproduction et traduction mzme partielle interdite.

Tous droits réservts potir lous pays

y compris i'U,R.S.S. et les pays scandinaves

ISBN 2-86433-016-4 


\title{
AVANT-PROPOS
}

L'opposition soulignée par Horace entre éthique et esthétique Aut prodesse volunt aut delectare poetae - que les Elisabéthains aiment à évoquer, pourrait être le lien qui rattache les unes aux autres les communications, rencontres et table ronde de notre troisième congrès (1981) sur le thème : "Théátre et Idéologies: Marlowe, Shakespeare.» Toutefois, le résultat de nos échanges montre un dépassement possible de l'alternative éthique / esthétique, puisqu'il met en valeur l'insertion d'idéologies susceptibles de transformer la fonction même de la littérature.

Les pièces analysées dans le contexte de leur temps révèlent de multiples aspects de la vision du monde et de la place que l'homme $y$ occupe, tels que les projette le théâtre anglais au tournant du XVIe et au début du XVIIe siècle.

Une exploration du langage et de la pensée des deux grands dramaturges aboutit à un commentaire sur les courants philosophiques ou religieux, sur les idées parfois contradictoires et sur la curiosité intellectuelle jamais assouvie qui se font jour à travers la rhétorique de la scène et le jeu dramatique, du Faust de Marlowe à La Tempéte de Shakespeare.

\author{
M.T. jones-Davies
}

Présidente de la Société Française Shakespeare 
SOCIETE FRANCAISE SHAKESPEARE Actes de Congrès.1981

\section{THEATRE ET IDEOLOGIES : Marlowe, Shakespeare}

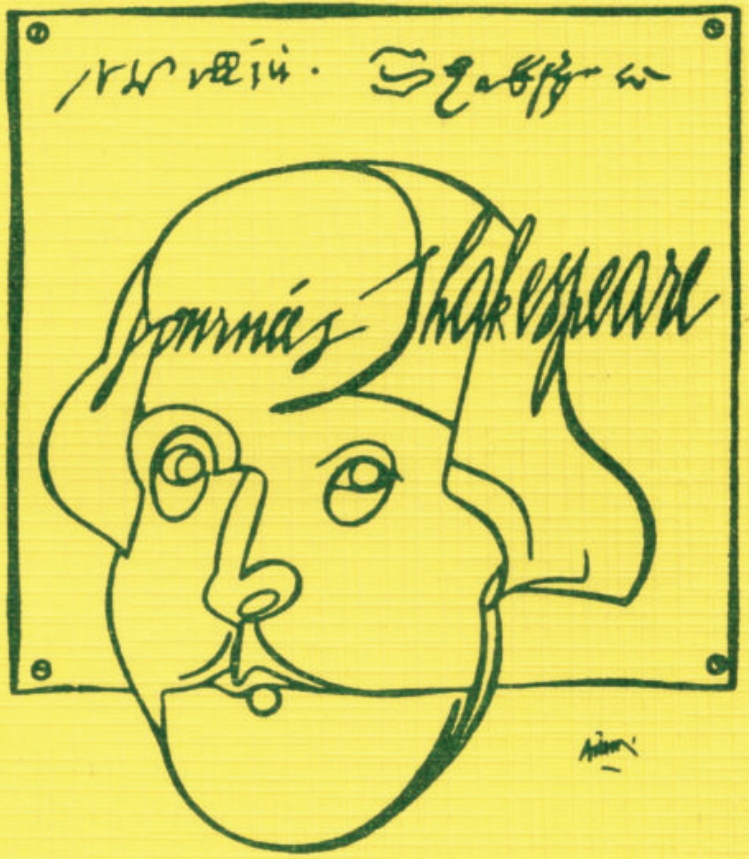

DIRECTEUR DE LA PUBLICATION M.T. Jones - Davies

JEAN TOUZOT Libraire - Editeur 38 , rue Saint-Sulpice 75278 PARIS CEDEX 061982 
Illustration de la couverture : affiche de Valerio Adami, spécialement conçue pour les Journées Shakespeare 1979 au Centre Georges Pompidou 


\section{TABLE DES MATIERES}

M.T. JONES-DAVIES Avant-propos

Théâtre et Idéologies: Marlowe, Shakespeare

S. TRUCHET

The Alchemy of Beauty: aesthetic principles in I Tamburlaine and their relationship to the hermetic ideology.

D. PRUDHOMME

L'idéologie du Juif de Malte et du Marchand de Venise.

D. GOY-BLANQUET

De Hall à Shakespeare : quelques glissements idéologiques opérés par la dramatisation dans Henry $I V$.

M.T. JONES-DAVIES Le Monıde du «si»: Idéologies Incertaines dans Comme il vous Plaira.

G. VENET

Temps et Idéologie : Marlowe, Shakespeare.

J. RICHER

Les Sept Caractères et les Quatre Tempéraments dans Jules César de Shakespeare.

F. LAROQUE

En marge de l'Idéologie : Antimasque et Grotesque dans le Dr Faustus et La Tempête.

A. LECERCLE-SWEET Conscience et Méconnaissance dans Edward The Second de Marlowe.

M.A. CONEJERO Shakespeare's Aesthe tic Scheme. (Extrait).

P.STEWART

Shylock, Shakespeare's Alien.

(Royal Shakespeare Company)

J.RUSSELL BROWN The Romantics' Shakespe are.

R. GILL

The Christian Ideology of Dr Faustus.

J. JACQUOT

Marlowe : de quelques problèmes d'interprétation. 
Rencontres entre Universitaires et Gens de Théâtre

Vendredi 4 décembre 1981
- Stuart Seide :Présentation du

Songe d'une nuit d'Eté. Mise en scène pour le Théâtre de Chaillot janvier 1982

- A propos d'Edouard (II) de

Bernard Turle

Bernard Turle : Métamorphoses de l'auteur et de l'écriture dramatiques. Intervention de Colin Harris (metteur en scène) 


\section{CONSCIENCE ET MECONNAISSANCE \\ DANS \\ EDWARD THE SECOND DE MARLOWE.}

En prenant un cas spécifique - l'écriture de l'histoire dans Edward the Second - nous avons voulu montrer l'imbrication étroite dans l'idéologie de composantes à la fois conscientes et inconscientes - et plus particulièrement l'enchevêtrement du réel, de l'imaginaire et du symbolique.

Pour mieux accorder notre discours à son objet, nous l'avons centré autour d'un topos, d'une figure, qui se trouve être la clé de voûte des trois domaines qui nous concernent - à savoir :

1. la tragédie comme genre

2. l'idéologie comme production d'une conscience méconnaissante

3. le fantasme comme discours censuré et scénario à éclipses.

Notre lecture se place en effet sous le signe de trois modes de renversement :

- le premier, philosophique, on le trouve au centre de l'Idéologie Allemande, où l'auteur recourt à l'analogie au sens kantien, dialectique, du terme - de la camera obscura pour décrire à la fois le processus de renversement qui se produit dans toute idéologie, et la nécessité de ce processus :

«Et, si, dans toute l'idéologie, les hommes et leurs rapports nous apparaissent placés la tête en bas comme dans une camera obscura, ce phénomène découle de leur processus de vie historique, absolument comme le renversement des objets sur la rétine découle de son processus de vie directement physique.»

Ce renversement est le premier logiquement, car il sous-tend implicitement les deux autres, centraux à notre argument.

le deuxième est littéraire. En effet, on peut dire qu'à travers ses défilés analytiques, notre lecture n'est pas autre chose qu'une espèce de retour à Aristote, plus exactement, à la peripeteia comme ressort sine qua non du texte tragique - en d'autres termes à ce que Jean-Pierre Vernant 
appelle

"ce pur schème opératoire de renversement qui est le modèle que la tragédie met en oeuvre sur tous les plans où elle se déploie».

et dont l'exemple le plus classique est la péripétie zetôn/ zetomenon qui se produit au cours de l'action d'OEdipe Roi, où c'est justement celui qui mène l'enquête (zetein). qui se renverse en son objet. Chercheur recherché, voilà la péripétie qui, pour Vernant, est le noyau irréductible à partir duquel le reste du texte s'engendre, et qui se retrouve jusque dans le titre: OEdipe : oida (savoir surhumain) qui se renverse en oidos pous (pied enflé) stigmate du paria bestial.

- le troisieme mode annoncé plus haut est constitué par ce «renversement dans le contraire» (Verkehrung ins Genenteil), où Freud voyait le blueprint des mécanismes de défense que la psyché humaine a su élaborer; noyau irréductible du processus de symbolisation à partir du célèbre scénario avec la bobine, «Fort !/Da !» (Va-t'en / Viens là) par lequel l'enfant exorcise sa dépendance à l'égard de son entourage. C'est ce renversement-là que l'on retrouve à un niveau bien plus sophistiqué dans la structure du fantasme, ce regard sans point de vue, sans sujet fixe, où celui qui regarde se renverse aussitôt en celui qui est regardé, et dont Maurice Merleau-- Ponty disait que

«voyant et visible se réciproquent [à tel point que] on ne sait plus qui voit et qui est vu : [et il ajoute] c'est cette Visibilité pour laquelle il n'y a pas de nom en philosophie traditionnelle».

Ce manque de nom, Jacques Lacan le comble en désignant cette réversibilité du voir par l'algorithme du fantasme qui se lit dans les deux sens - le sujet barré, le losange (trouée du regard), et l'objet, cause et effet du désir : \$১a.

Pour donner deux brefs exemples nearer to home de ce troisième mode de renversement, on peut montrer, nous semble-t-il, que le dumb-show dans Hamlet - reliquat pour certains, redondance pour d'autres - fonctionne en fait autant par rapport à la mise en scène qui le précéde immédiatement, et dont Hamlet et Ophélie sont les acteurs, que par rapport à The Murder of Gonzago, qui lui succède, où, cette fois, ce sont Hamlet et Ophélie qui se sont renversés en spectateurs, et spectateurs justement de ceux qui, dans la scène précédente, les regardaient des coulisses -- à 
savoir Claudius et Polonius. On peut montrer encore que, dans la folie d'Ophélie, une composante capitale est le renversement du discours du manque - et notamment de la défloration (qui culmine dans la chanson du Valentine) en une gestualité qui consiste précisément à tout couvrir de fleurs, et qui a ceci d'excessif, qu'elle mène tout droit à la mort. Ce renversement-là est d'ailleurs annoncé dès l'apparition d'Ophélie folle par le mythe du hibou et du Christ, ou le manque (le bout de pâte que la fille avare enlève au pain destiné à Jésus) se renverse en un pain qui gonfle, à tel point que l'horreur lui fait pousser les hoo, hoo, hoo du hibou. Est-il besoin de préciser le rôle analogue joué par "the dough which immediately began to swell, and presently became of a most enormous size", provoquant la métamorphose de la fille du boulanger en oiseau de proie, et les «long purples / That illiberal shepherds give a grosser name», qui, peu après, précipitent Ophelia to a watery death ? Renversement du fantasme dans le premier cas, renversement du symptôme dans le second.

Mais laissons là Hamlet pour Edward the Second, et notamment pour la contrée largement «undiscover'd» sinon «unknown», qu'est cet au-delà spécifiquement théâtral dénommé par Jean Gillibert «l'arrière-temps» du spectacle :

Il y a un geste ou un acte - un acteur - qui produit une action absolument désintéressée (et en cela Aristote, Kant et Artaud ont dit la même chose) et l'arrière-plan d'un acte; arrière-plan qui efface l'acteur derrière le spectateur, le public derrière le privé, l'exhibition derrière l'inhibition; [... l'acte [...] agrandit démesurément les limites du corps, fait sortir du rêve celui qui est agi, mais plonge dans le rêve celui qui «subit» l'acte (le spectateur) [...] ceci s'étant accompagné d'un arrière-temps, de coulisse, de souterrain, d'enfer".

C'est qu'il nous a semblé que l'économie interne d'Edouard II avait été quelque peu négligée par les critiques, souvent plus préoccupés par le contexte - proche ou lointain - que par le texte; ce qui, après tout, est leur droit. Pour renverser la tendance, nous nous sommes inspirée du principe énoncé par Jean-Pierre Richard dans l'introduction à ses admirables Microlectures : 
«Il m'a semblé [...] à l'expérience, qu'à partir du plus petit, c'était le plus précieux, en tout cas le plus singulier d'une oeuvre qui pourrait être lu et dit.»

Pour accéder à cet «enfer», nous nous sommes ainsi penchée, entre autres, sur deux aspects - microphénomènes dirait Richard - de la pièce, dont nous ne sachions pas qu'ils aient été abordés ailleurs, et à partir desquels, nous semble$t$-il, on peut utilement éclairer l'ensemble de l'oeuvre.

Le premier de ces aspects c'est l'instance de la lettre ou le texte dans le texte.

En entendant les quatre premiers mots du spectacle, de la première lettre, celle qu'Edouard envoie à Gaveston au lever du rideau - quel analyste n'aurait pas l'impression de rêver ? Voilà, planté d'entrée de jeu et sans ambages, le signe brut sine qua non du fait tragique - le père «mort»:

My father is deceased

Rappelons la division originaire :

Dans la comédie attique, on montre et on circonscrit les pouvoirs phalliques [...] Le phallus en cuir bouilli dont s'armaient les acteurs de comédie était le vrai sujet de la réunion. On châtrait le phallus, on le lutinait aussi, on se moquait de l'érection, on la travestissait (cf. Aristophane) pour conjurer ses pouvoirs apotropaïques. La tragédie se réservait de «voiler» le phallus - sous le signe du "père mort»; le phallus doit ressusciter dans la lignée : phallus d'outre-tombe. Il ne faut pas que la race (humaine) meure ! Conjurons inceste, cannibalisme, parricide.

Ainsi, dans la pièce de Marlowe, le père est bel et bien mort, mais comme il s'avère peu après (on se prépare à célébrer la messe des morts), il n'est pas encore enterré. Or dans ce hiatus entre mort physique et vie éternelle, il reste le temps du sacrilège qui ne manque pas - par métonymie de se produire. En effet, s'il n'y a pas parricide, il y a profanation : sur la personne du double spirituel du roi défunt, celui qui précisément avait interdit et banni l'objet du désir "contre nature», et qui a présent s'apprête à organiser les funérailles d'Edouard ler : l'évêque de Coventry. Marlowe ici se conforme rigoureusement aux quelques notations laconiques de Holinshed où l'élément de transgression apparaît déjà clairement : «alors que, trois jours après la mort d'Edouard ler, 
Walter Langton, évêque de Coventry [...] allait à Westminster prendre les dispositions pour l'enterrement, il fut saisi sur l'ordre du nouveau roi [...] et fut aussitôt livré à ce Peers [Gaveston]»

Dans le premier couplet - capital - de l'oeuvre, on pourrait dire en effet que le silence central (la césure) "assourdissant» dans son éloquence, constitue presque un parricide «après coup» :

My father is deceased. Come Gaveston

And share the kingdom with thy dearest friend.

Ainsi, cette première lettre fait d'emblée surgir à travers le désir homosexuel l'occultation du père porteur du phallus symbolique de la loi. Et il faut bien le dire, le Nom de ce Père-là était particulièrement significatif, car il est entré dans l'histoire pour ainsi dire par synecdoque, sous le sobriquet affectueux, commenté dans la pièce, d'Edouard «Longues Jambes» (Longshanks) - distingué par la taille démesurée de ses membres inférieurs! Quoi qu'il en soit, l'ordre symbolique, dans ses deux branches principales - la temporelle et la spirituelle - est d'entrée de jeu occulté dans la pièce.

La conséquence est explicitement formulée dès le deuxième vers de cette lettre distique. Quand l'instance symbolique vient à tomber, c'est l'imaginaire qui reprend ses droits. Or le propre de l'imaginaire, comme l'on sait, c'est d'une part la labilité des objets (partiels) qui le composent, de l'autre la Spaltung, le clivage, qui structure le sujet à travers ses objets. C'est cette intuition liminaire qui fonde la cohérence profonde de la vision de Marlowe dans Edouard II. C'est pourquoi, du pere mort du premiers vers, l'on passe directement au clivage annoncé dès le second; nous voulons parler du partage du royaume aussitôt propose par le roi à son alter ego : «share the kingdom with thy dearest friend». Clivage qui va bientôt s'aggraver, d'ailleurs, et devenir morcellement, quand le roi réagit à la menace de perdre son mignon en proposant aux barons de diviser le royaume entre eux.

Ainsi Marlowe commence à mettre en place une série d'équivalences parfaites:

1. entre guerre civile et Ichspaltung, cette refente du sujet dont Marlowe fournit une définition admirable :

thou from this land/I from myself am banish'd ; 
2. entre mutability et labilité fortement accrue par le fait du narcissisme qui fonde l'homosexualité, et une mutability particulièrement prononcée sous la dynastie des trois premiers Edouard, où le royaume se trouve dans un état exceptionnel de flux entre les principales composantes sociales du pays : le système féodal étant en ruines alors que le système d'une monarchie absolue, comme celui des Tudors, n'existait pas encore.

3. si bien que cette "situation d'entre-deux", par laquelle Pontalis définit la bisexualité, bisexualité inhérente aux rapports entre Edouard et son Protée d'amant - ( «as if that Proteus, god of Shapes, appear'd» dit Mortimer de Gaveston) - s'applique tout autant à l'Angleterre des Plantagenets dont Trevelyan écrit qu'elle est essentiellement "déchirée par cette lutte permanente entre les Barons et le Roi», - Proteus étant bien évidemment la représentation paroxystique, au niveau du simple individu, de ce que c'est que la mutability humaine dans la polis sociale

De cette manière, grâce à ce triple jeu de parallèles, Marlowe dans cette pièce renouvelle l'exploit de Sophocle dans OEdipe Roi : à savoir il réussit à créer à tous les niveaux et jusque dans les détails un parfait jeu de miroirs entre inversion sexuelle et inversion sociale, - en d'autres termes, entre amours contre nature et troubles contre nature dans le body politic. Or, pour rendre parfait ce jeu de miroirs, Marlowe a dû passer par la réécriture de l'histoire : en effet les objets du désir du roi sont tous présentés dans la pièce soit comme des "paysans», soit comme des "parvenus», alors que les Despencer faisaient partie d'une famille des plus respectables, (dont Diana, future reine d'Angleterre, sera sans doute le plus illustre rejeton); Sir Robert Baldock était même le Lord Chancellor d'Edouard, et Gaveston surtout était loin d'être aussi base born que les barons voudraient le faire croire "Thou villain that hardly art a gentleman by birth" dit Mortimer, alors que Holinshed le décrit comme «un gentilhomme de Gascogne” (an esquire of Gascoine). Notons que Brecht, dans sa réécriture de la pièce ne fait que finir le travail que Marlowe avait commencé : là il est devenu «fils de boucher», on s'y réfère constamment à lui comme «dieser Schlächtersohn».

Or, cette distorsion opérée par Marlowe a pour 
fonction de créer un lien nécessaire, et en dernière analyse tragique - marqué par l'ananké - entre le choix d'objet sexuel, et les basses origines de ce dernier. Car ce n'est pas seulement qu'un "paysan» soit placé sur le trône de l'Angleterre, c'est qu'il est placé sur le trône de la Reine; d'où la pointe à deux faces du couplet ironique :

Your grace doth well to place him by your side,

For nowhere else the new earl is so safe.»

De même, dans OEdipe Roi, ce n'est pas seulement qu'un pharmakos soit élu roi "égal aux dieux», devienne iso theos, mais que, du même coup, un parricide entre dans le lit de sa mère.

Bref, on pourrait presque dire que le «premier couplet d'Edouard II 》 contient à lui seul les germes de la tragédie entière. C'est pourquoi il est détaché dans le texte par les italiques : italiques auxquels font pendant deux autres vers ainsi imprimés, qui surgissent en position symétriquement inverse, tout à fait à la fin de la pièce. Nous voulons parler de ce message mortifère, essentiellement clivable, selon que l'on y introduit une césure ou non :

"Edwardum occidere nolite timere bonum est"

Il s'agit dans les deux cas de lettres : celle du début est provoquée par la mort d'un roi; celle de la fin provoque la mort d'un roi, - péripétie aristotélicienne à l'état brut. Or cette dernière lettre prouve, si besoin était, que dans cette pièce, l'ordre symbolique - ici langagier - est irrémédiablement battu en brèche par le clivage du signifiant et la labilité du sens - autrement dit par cette amphibologie dont Vernant fait le hallmark du texte tragique : dans ce cas précis - «Craignez de tuer le roi / il est bon de le tuer».

Il faut ajouter à ces deux lettres symétriques et complémentaires une troisième, qui, elle aussi, est placée sous le signe du clivage : ici dans un sens tout à fait concret. C'est celle qu'Edouard déchire de manière à couper en deux le nom qu'elle porte en signature - celle de Mortimer :

By Mortimer, whose name is written here!

Well may I rent his name that rends my heart.

L'étymologie est ici très éclairante; en effet, l'anglais rent vient de la racine sanscrite (randhra) qui veut dire «fissure, fente», en anglais «split». Bref, il ne s'agit pas de n'importe quelle mise en morceaux mais de la configuration méme dont il est question ici /splitting / Spaltung : clivage, 
ou refente). Ce qui est primordial pourtant, c'est de voir comment le clivage du nom se retourne contre celui quil'a effectué. L'intuition de Marlowe touche ici véritablement au génie, car en clivant le signifiant «Mortimer» en ses deux parties constituantes - déjà commentées au cours de l'action par ce fils de croisé qui le porte : MER + MORTE ...

This tatter'd ensign of my ancestors, Which swept the desert shore of that Dead Sea

Whereof we got the name of Mortimer.

... Edouard produit la forme spécifique que prendra la dernière étape de son calvaire : à savoir cette mer que les geôliers appellent un lake. Car la Mer Morte est un lac, a mere. D'où ce vers, étrange à première vue, de Matrevis, quand il fait entrer Lightborn au cachot : «Here is the keys, and this is the lake.» Mer de mort, faite de la décomposition de choses mortes, mais aussi mortifère, provoquant la mort :"so to make an end of him. with the abominable stench thereof», lit-on dans Holinshed.

Or, ce renversement (MORTIMER -MER DE MORT). péripétie en forme de chiasme, comme on n'espère plus guère en trouver en dehors des auteurs grecs, Marlowe l'a construit, là aussi, en réécrivant l'histoire. En effet, si le dernier cercle de l'enfer - celui ou préside Lucifer / Lightborn (e) en personne - prend l'aspect du cloacum mundi - que Brecht n'a fait que rendre explicite en le transférant de Berkeley à la Tour de Londres, et carrément au «Kloake des Turms, wo fällt das Kot Londons» (où tombe l'excrément de la cité) - ou, comme le dit le suppiicié lui-même :

Th is dungeon where they keep me is the sink,

Wherein the filth of all the castle falls,

c'est qu'il n'y avait absolument rien de tel dans les sources, ni dans les Annales de Stow, ni dans les Chroniques de Holinshed. Au moment où on est venu l'emmener au supplice, en effet, nous lisons dans les deux cas que le roi était (je cite) "profondément endormi dans son lit»"

they came sodenly one night into the chamber where he ley in bed fast asleepe, and with heavie feather beddes (or a table as some write) being cast upon him, they kept him downe, and withall put into his fundament an horne, and through the same they thrust up into his bodie a hotte 
spit.

Pour nous, cette péripétie chiastique est un des exemples les plus importants où le génie du dramaturge, à travers l'intuition l'Einfühlung proprement tragique, subvertit la représentation de l'histoire par le travail de l'inconscient. Et cela en fonction de l'isomorphisme des trois modes de renversement définis au début.

La quatrieme lettre est celle que Gaveston écrit à sa future épouse - variante ironique - qui complète cette constellation symptomatique. Il s'agit cette fois non d'une annonce de mort, mais d'une déclaration d'amour. Ainsi, le sort qui lui est réservé n'est pas d'être détruite en subissant un clivage, mais, bien au contraire, d'être conservée grâce au sens que le terme cleavage a gardé en anglais contemporain. clivage bien réel celui là, à savoir l'espace entre les seins d'une dame - cachette traditionnelle pour de pareilles missives :

Niece : «Puts the letter into her bosom.»

Cette lettre écrite par Gaveston à sa fiancée fait pendant à celle qu'il lit au lever du rideau. Membres galants du carré épistolaire, ils n'en recèlent pas moins que les deux autres les germes de la mort - dans ce cas précis, celle affectée d'un diminutif précieux, euphémique, la «petite» mort du double entendre contenu dans le vers qu'elle cite : «I will not be long from thee, though I die».

Ainsi, dans une oeuvre toute en heurts ou accouplements «contre nature», la lettre, symbole de l'échange, se place ainsi sur la ligne de partage entre Eros et Thanatos. La lettre, missive ou signifiant, montre de façon saisissante que s'il y a quelque chose de pourri dans le royaume d'Angleterre, c'est que l'ordre symbolique - dans son instance privilégiée, le logos - est subverti, miné dès le départ par cette Spaltung qui est le propre de l'imaginaire. Or, ce premier symptôme s'accompagne du phénomène complémentaire - l'autre face de la médaille (plus exactement du médaillon), car roi et mignon ne manquent pas d'échanger leurs «images» (exchange portraits) en se séparant.

En effet, s'il y a prolifération de clivages, il y a inversement toute une problématique du double. Dans cet univers spéculaire, comment l'expiation - mise à mort anale - pourrait-elle être autre chose que l'image de la 
transgression de sexualité anale ? C'est donc dans le cloaque du macrocosme qu'elle a lieu, ce "cesspool of the universe», dont parle Tillyard à propos de The Mirror of the World. Du coup, les premiers mots que Lightborn / Lucifer adresse au roi sont-ils le double, la parodie diabolique, des mots de l'archange Gabriel lors de l'Annonciation : «I come to bring you tidings of great joy». Du moins c'est comme ça que nous les entendons, car que dit Lightborn? you, and bring you joyful news.) Dans l'élévation momentanée du style, le drame religieux se profile à travers la tragédie historique; la forme ancienne apparaít comme la tête en bas, la formule sacrée véhicule maintenant un contenu politique, et l'annonce de vie éternelle se renverse en annonce de mort subite, assassinat machiavélique.

D'ailleurs, Lightborn est lui-même le double nocturne, infernal, de la première vision de la mort qui nous est présentée dans la pièce. Nous voulons parler du sombre (gloomy) moissonneur, qui surgit des plaines fertiles du Gloucestershire ou de la riche vallée de la Wye pour venir dénoncer Edouard à l'Abbaye de Neath. Cette figure-là représentait la mort sous son aspect «naturel» - c'est -àdire intégrée au cycle de la nature. Or le cercle de l'enfer est la parodie du cycle de la nature.

$\mathrm{Ce}$ ne sont là que quelques exemples ponctuels et rapides, mais en dernière analyse la portée de ce thème va bien plus loin - jusqu'à son expression extrême : la coincidentia oppositorum. C'est que, dans cette pièce, comme s'en indigne le sévère baron Lancaster, une chose et son contraire peuvent être la même chose. D'où tous ces mouvements de refus et d'acceptation, d'aller et de retour, de départ et d'arrivée, qui scandent l'action : bannissement / rappel de Gaveston, départ pour l'Irlande /retour d'Irlande au début de l'oeuvre; le déplacement répété du roi de Killingworth à Berkeley et vice versa à la fin; ou, tout au long du spectacle, les tours de girouette de Kent, pour ne citer que les cas les plus flagrants. Tous ces revirements brusques ne sont pas tant le signe d'une psychologie fruste de la part de Marlowe ou d'une dramaturgie archaique : «épisodic, unchoate» trouve-t-on sous la plume des critiques Ils sont au contraire l'indice éclatant du relâchement des instances du symbolique qui entraîne cette labilité déjà évoquée, mais plus précisément ces Verkehrugen ins 
Gegenteil (renversements dans le contraire) par quoi la Spaltung se donne à reconnaître comme telle. Ce n'est pas un hasard si c'est dans la bouche de celui qui fut de loin le plus redoutable parce que le plus titré des barons (Lancaster, Derby, Salisbury, Lincoln, Leicester) que Marlowe met cette condamnation du règne de la coincidentia oppositorum. En effet, ainsi pourvu des emblèmes de cette puissance physique ( «il a plus de fief's qu'un âne ne saurait porter») et symbolique (démultiplication des noms) sans égal parmi l'aristocratie, il devient tout naturellement le maître de vérité, l'Autre, celui qui se place au-dessus de ces jeux de miroirs où le même et l'autre se renversent et se rejoignent. C'est pourquoi il serait difficile de surestimer la portée de ses paroles quand il dit :

Lan: Fie Mortimer, dishonour not thyself !

Can this be true, twas good to banish him ?

And is this true, to call him home again?

Such reasons make white black, and dark night

day,

Mor.: My lord of Lancaster, mark the respect.

Lan.: In no respect can contraries be true.

Ce n'est pas là une simple remontrance prononcée par l'un quelconque des barons, mais par celui qu'on appelle l'«Inexorable»: très précisément, le représentant de l'instance de la Loi, «immuable aux prières» (in-ex-orarae). Nous avons là le point nodal, la matrice de la tragédie, énoncés par celui qui tient en quelque sorte le rôle du Tirésias de la cour d'Angleterre. Notons que Warwick, le «Brutal» (rough). celui qui reprend et fait décapiter Gaveston, représente la face spécifiquement castatrice de cette instance; et, de même qu'Edouard Ier est resté dans la mémoire collective par la longueur excessive de ses membres inférieurs (Longshanks). inversement le membre supérieur de Warwick (sa tête coupée) est destiné à surplomber toutes les autres : thy head shall over looke the rest»(«ta tête dépassera les autres"). Si bien qu'en les faisant décapiter tous les deux d'un seul coup, il tue ce Père - à deux faces une deuxième fois, et, du même coup, instaure le règne du deuxième mignon - monstre à deux têtes lui aussi, ayant pour nom Spenser et Baldock.

On peut dire que les mots de Lancaster ( «In no respect can contraries be true») découlent directement du premier 
couplet de la pièce. En occultant le père porteur du phallus symbolique de la loi, le désir homosexuel évite la castration: résultat, l'amphibologie s'installe dans la langue, les «renversements dans le contraire» dans la représentation, car ce n'est pas autre chose que Lancaster exprime ici. Tout le programme de l'oeuvre y est annoncé, y compris le dénouement tragique. Car chassez le naturel, il revient au galop. Or, l'expiation d'Edouard n'a jamais été surpassée comme représentation de la revanche du phallus - cette broche chauffée à blanc, plongée dans l'anus et «tournée dans les deux sens». Comme dans la tragédie de Sophocle, ce sont justement les contraires qui sont vrais, OEdipe étant à la fois oida (je sais), d'une omniscience qui confond la Sphinge, et oidos pous («pied enflé») celui qui ne sait absolument rien - de lui-même. Et de même que, grâce à l'inceste (non à l'homosexualité), OEdipe évite la castration, produisant cette prolifération d'amphibologies et de renversements relevés par Jean-Pierre Vernant dans son admirable essai sur cette oeuvre, de même les orbites sanglantes du tableau final signifient la même revanche du signifiant phallique préalablement forclos.

Si nous considérons ce qu'on peut appeler le prologue (où Gaveston reste seul après le lever du rideau), on peut mieux percevoir la rigueur du procédé de Marlowe : en effet, deux opérations inverses et complémentaires s'y succèdent. Dans son premier discours, Gaveston insiste tout au long sur les phénomènes de clivage: dans l'ordre .

1. le partage territorial du royaume.

2. la faille océanographique entre la France et l'Angleterre (la Manche jouant le rôle de l'Hellespont séparant Hero et Leandre).

3. le clivage cosmologique entre Londres et Elysium.

4. la division politique et ontologique entre le roi et le peuple.

5. le partage chronologique entre le jour et la nuit (absence / présence du soleil.)

6. la division géographique entre l'Europe du Nord et l'Europe du Sud (Arctic/ Angleterre).

7. le hiatus social entre les pairs du royaume et les pauvres du royaume.

C'est l'opération inverse qu'effectue le second long discours du même personnage peu après. En brossant le 
tableau de divertissements équivoques, ce discours, en effet, s'emploie à brouiller les principaux paramètres qu'il met en oeuvre : Dieu).

1. sexuels d'abord (travestissement-bisexualité)

2. ontologiques ensuite (le satyre : bête/homme/

3. sociaux (la fête, où acteurs et spectateurs, nobles et «ignobles» au sens étymologique se mêlent et se confondent).

4. syntaxiques enfin (actif/passif : chasseur chasse le my the d'Actéon déchiré vivant par ses chiens).

Pourtant, de toutes les métamorphoses orchestrées par ce "Proteus», la plus importante est celle qui, implicitement, structure non seulement ce discours dans son ensemble, mais la pièce tout entière : celle d'une sensualité démesurée, en un sadisme sans bornes.

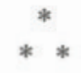

Nous nous sommes concentrée jusqu'ici essentiellement sur la subversion du logos, dans son instance privilégiée, la lettre - c'est-à-dire sur le domaine du symbolique. Or, ce qui se passe sur le plan du symbolique a son répondant dans l'imaginaire. En effet, peut-être le véritable trait de génie du travail élaboratif de Marlowe, c'est le renversement progressif mais inéluctable que subit cet élément omniprésent dans la pièce, l'eau, et plus spécialement l'image de la grande absente de la configuration homosexuelle - la mère. Dans un article intitulé "L'Insaisissable Entre-Deux», qui est un commentaire sur la naissance d'Hermaphrodite, J.B. Pontalis évoque le rôle primordial de "cette eau féminine dont la transparence passe sous nos yeux au sombre de l'eau funèbre». En effet, il est fascinant de voir comment, au fil de l'action d'Edouard II, les couleurs et les connotations de l'eau petit à petit se retournent en leur contraire. Disons tout d'abord que dans peu de pièces on a autant l'impression d'une action qui se déroule sur une île, dans cette Angleterre "set in a silver sea» dont parle John of Gaunt dans Richard II.

Au Sud, la Manche, d'où Gaveston débarque au lever du rideau; à l'est, le Pas-de-Calais, séparant l'Angle terre du 
Hairaut (Belgique), traversé par la reine et son armée; au nord-est, la mer du Nord, et le petit port de Scarborough, néfaste au premier mignon du roi; enfin au sud-ouest (symétriquement inverse) la mer d'Irlande, traversée et retraversée par Gaveston, ainsi que le grand port de Bristol, néfaste au roi et à son second mignon, Spencer. Ainsi, au cours de la pièce, on fait un peu le tour de l'île en bateau : la mer constitue en quelque sorte sinon la clôture géographique, du moins la circonférence concrète du champ de l'action, limite perceptible des mouvements centrifuges et centripètes qui scandent l'évolution des événements.

C'est donc par un coup de génie que, dans la tragédie du pharmakos, de la mise à mort du tyran, Marlowe renverse complètement cette image : en effet, comme nous l'avons vu, la Mer Morte du cloacum mundi est au centre du royaume, où elle entoure le roi, stagnante et fétide. Ce renversement-là, pourtant, ne reste pas une pure abstraction Sur le canevas ainsi mis en place, Marlowe passe des plus riantes transparences aux tons les plus sombres de cette "eau funèbre» dont parle Pontalis.

Il faut aussitôt préciser pourtant que la mort qui, dans le mythe-matrice de Narcisse, surgit précisément de la belle transparence des eaux de l'étang, n'est jamais loin dans ce courant souterrain qui traverse l'oeuvre.

Ainsi, si l'Hellespont évoqué par Gaveston, est associé dans un premier temps aux joies de l'amour triomphant, elle finit néanmoins par engloutir Léandre. Même jeu dans le mythe d'Hylas, évoqué peu après par Edouard, et étonnamment proche du mythe de la naissance d'Hermaphrodite : en effet, cet amant d'Hercule, désiré par les nymphes, est lui aussi resté prisonnier des eaux. D'autre part, ce n'est pas du tout par hasard que dans le discours "satyrique" de Gaveston, toute la scène est conçue en termes de jeux essentiellement aquatiques, ce qui permet à Marlowe de la terminer par l'épisode où un Actéon voyeur, observant Diane au bain, périt de ce fait dans «a lake of gore».

Par ailleurs, il est remarquable que par deux reprises, ce soit en se servant de la relation mer/ile que le roi exprime son refus - son incapacité - de se séparer de son favori. La première fois, plutôt que de renoncer à ce dernier, il invite les flots à venir submerger son île. La seconde fois - comme 
l'on pourrait s'y attendre - l'image est symétriquement inversée : c'est alors son île qu'il condamne à «voguer sur les ocẻans»:

Ere my sweet Gaveston shall part from me,

This isle shall fleet upon the ocean,

And wander to the unf requented Inde.

C'est cette association constante entre l'élément aquatique et le personnage bisexuel qui fait qu'à plusieurs reprises on se réfère à Gaveston comme à un poisson. Et ici encore - protéité oblige - l'image est clivée et réversible: tantôt il est poisson torpille destructeur (vile torpedo), tantôt il est poisson volant détruit par les oiseaux : «a flying-fish/Which ... takes the air/No sooner it is up, but there's a fowl/that seizeth it».

Et Edouard de poursuivre dans la même veine en interdisant aux "plus gigantesques monstres marins" de l'engloutir. Parole dangereuse, car c'est justement parce qu'il a confié son ami aux flots ("gone by water»), au lieu de l'emmener avec lui par terre, que Gaveston est pris.

Un renversement particulièrement important est celui qui affecte l'eau (infiniment moins bleue et moins pure que les eaux grecques) du ruisseau britannique : «channel water». Dans la première scène de rencontre entre Edouard et son mignon, cette eau doit jouer le rôle - sacrilège d'eau de baptême, pour "rebaptiser» l'évêque de Coventry, comme l'ordonne Gaveston. Inversement, dans l'avantdernier cercle de l'enfer, cette même eau («channel water») doit servir un but diamétralement opposé : pour faire la toilette funèbre du futur supplicié - en lui rasant la barbe.

Bref, peu à peu, l'image de l'eau passe de la pureté et de la transparence pour aboutir à cette version marlovienne - spécifiquement aquatique - de l'enfer, où le pécheur doit confronter Lucifer/Lightborn, qui, lui-même, disparaît aussitôt dans les eaux "extérieures» du château : c'est-à-dire dans la douve qui l'entoure - autre retouche marlovienne à I'histoire : ("Come let us cast the body in the moat»).

Que doit-on conclure de ce renversement ? dans cette pièce, le père, quoique mort, n'est pas absent : l'ombre du puissant "Longues Jambes" est encore "vivant dans l'esprit du peuple», dit Mortimer, comme représentant d'un ordre moral et institutionnel tombé en déchéance. Il vit aussi dans ce que l'on pourrait appeler sans exagération le double 
positif du roi, le «bon» fils. En effet, Edouard Ier (ainsi que Simon de Montfort) a toujours existé dans la mémoire collective des Anglais comme Father of the English Parliament, celui qui, entre tous, a érigé le logos en institution de Loi, transcendant luttes et rivalités, dans le macrocosme social, - ce qui ne fait que reproduire le rôle du Père tout court dans le microcosme de la famille. Ce n'est donc pas par hasard si Edouard II crée un clivage radical entre une parole "bonne», la parole parlementaire qui s'appelle justement parley et une parole «mauvaise», les «doux discours» (sweet speeches) et les «mensonges à table» (lies at dinner-time) en lesquels le favori du roi est passé maître. Un incident entièrement de l'invention de Marlowe, et qui rappelle étrangement le cas d'OEdipe, est très significatif à cet égard. Au lever du rideau, le père (Laios/Edouard Ier) est déjà mort. A l'entrée de la capitale du royaume (Thèbes/Londres) d'où il avait été exclu parce qu'il représentait un trop grand danger pour le pays, le paria, revenu de son exil (Gaveston/oidos pous), doit subir une épreuve orale tripartite. L'énigme que posait le Sphinx était une sorte de hors-d'oeuvre au repas cannibal qui suivait en cas d'échec, OEdipe est le seul à triompher de cette parole-piège. Dans la tragédie marlovienne, Gaveston fait triompher la parole-piège, ces lies at dinner-time que lui racontera le voyageur (le seul des trois qu'il engage), lors des agapes à la cour de Londres, - entrements plutôt que hors-d'oeuvre, pourrait-on dire.

Ce qu'il faut retenir, en tout cas, c'est l'association essentielle au sein de cette deuxième parole entre parler et manger - association qui fait de cette sorte de parole «mauvaise» - parce que régressive, oralité archaïque, placée non pas sous l'égide du Père, mais bien plutôt sous le signe du leurre (lies) qui caractérise l'image de l'autre originaire, c'est-à-dire maternel.

$\mathrm{Si}$ «le phallus d'outre-tombe ressuscite» à la fin de la tragédie, c'est précisément parce que, après une longue occultation par les jeux de miroir et les leurres du narcissisme, il finit par l'emporter sur le "mauvais» fils et ses émules tyranniques - très exactement quand le jeune prince va quérir la protection du Conseil contre Mortimer. Car ce fut par un acte de Parlement qu'Edouard fut destitué, et si Marlowe escamote ce détail, c'est justement 
pour nous laisser sur une sanglante image de décapitation/ castration, où la résurgence phallique balaie la fidélité historique.

La mère, elle, est absente. Pourtant, mer, mere ou lake, elle est d'autant plus présente sur le plan du fantasme. Car si le calvaire d'Edouard a pour fonction de le dépouiller progressivement de ses attributs -

d'abord royaux

puis virils

puis simplement humains
: sa couronne

: sa barbe

: ses membres, croupissant dans les eaux

- "And whether I have limbs or no I know not»-c'est que, avant de le livrer à l'épée, la mer (e), cette eau féminine dont parle Pontalis, finit elle aussi par prendre sa revanche. En effet, non seulement elle envahit jusqu'au centre symbolique du royaume («Where is the court but here? Here is the king") mais, ce faisant, elle fait du roi un embryon : "Sans crown, sans beard, sans limbs - sans everything», pour parodier Jaques. Car l'eau funèbre de Pontalis est aussi l'eau des origines. C'est ce double renversement - eau féminine, eau funèbre, eau des origines qui est la pierre angulaire de l'imaginaire dans la pièce de Marlowe.

Dans Edouard II, le souterrain, l'enfer du texte, est le lieu privilégié où l'histoire se réécrit. Cet ensemble de retouches et de travail élaboratif permet à Marlowe d'enchaîner les renversements à tous les niveaux de la pièce. Aussi devient-il le lieu par excellence de la subversion où, derrière la scène historique, se profile l'autre scène, anderes Schauspiel, celle du désir, et la conscience se démasque comme méconnaissance.

Ann LECERCLE-SWEET 